\title{
Neural network modelling reveals changes in directional connectivity between cortical and hypothalamic regions with increased BMI
}

\author{
Katharina Voigt ${ }^{1}$, Adeel Razi ${ }^{1,2,3,4}$, lan H. Harding $\mathbb{D}^{5,6}$, Zane B. Andrews $\mathbb{D}^{7}$ and Antonio Verdejo-Garcia (D) ${ }^{1 凶}$ \\ (c) The Author(s), under exclusive licence to Springer Nature Limited 2021
}

BACKGROUND/OBJECTIVES: Obesity has been ascribed to corticostriatal regions taking control over homeostatic areas. To test this assumption, we applied an effective connectivity approach to reveal the direction of information flow between brain regions and the valence of connections (excitatory versus inhibitory) as a function of increased BMI and homeostatic state.

SUBJECTS/METHODS: Forty-one participants (21 overweight/obese) underwent two resting-state fMRI scans: after overnight fasting (hunger) and following a standardised meal (satiety). We used spectral dynamic causal modelling to unravel hunger and increased BMI-related changes in directed connectivity between cortical, insular, striatal and hypothalamic regions.

RESULTS: During hunger, as compared to satiety, we found increased excitation of the ventromedial prefrontal cortex over the ventral striatum and hypothalamus, suggesting enhanced top-down modulation compensating energy depletion. Increased BMI was associated with increased excitation of the anterior insula over the hypothalamus across the hunger and satiety conditions. The interaction of hunger and increased BMI yielded decreased intra-cortical excitation from the dorso-lateral to the ventromedial prefrontal cortex.

CONCLUSIONS: Our findings suggest that excess weight and obesity is associated with persistent top-down excitation of the hypothalamus, regardless of homeostatic state, and hunger-related reductions of dorso-lateral to ventromedial prefrontal inputs. These findings are compatible with eating without hunger and reduced self-regulation views of obesity.

International Journal of Obesity (2021) 45:2447-2454; https://doi.org/10.1038/s41366-021-00918-y

\section{INTRODUCTION}

Obesity contributes to $\sim 5 \%$ of deaths globally, reduces life expectancy by almost a decade [1] and accounts for a $2.8 \%$ loss to the global gross domestic product [2]. The prevalence of obesity has risen in parallel with increased access to energy-dense, highly attractive foods [3]. These food choices can overtake the neural mechanisms that regulate homeostatic-based eating and promote overconsumption [4]. Obesity is underpinned by abnormal interactions between homeostatic and non-homeostatic brain systems. The homeostatic system regulates eating based on energy needs and is associated with hypothalamus activity [5]. The hedonic system encodes non-homeostatic aspects of food, such as incentive salience and affective value and involves striatal, insular and medial prefrontal regions [6]. Three main neurobiological theories of obesity have been suggested: (i) the 'hedonic eating' model proposes that brain areas processing reward (e.g., striatum, medial prefrontal cortex) become dominant over those involved in energy homeostasis (e.g., hypothalamus) in response to food cues
[5-7]; (ii) the 'eating without hunger' framework suggests that the neural regions processing salient external stimuli outweigh those involved in internal energy sensing [8]; and (iii) the 'self-regulation' view emphasises impaired top-down regulation of lower-level striatal and limbic regions [9, 10]. Understanding the interaction between the neural systems pinpointed by these theories is key to envisage new approaches to prevent and treat obesity.

Neuroimaging functional connectivity studies have the potential to improve our understanding of the interplay between homeostatic and non-homeostatic neural systems. Food choices activate brain regions involved in energy regulation (hypothalamus), interoception and inner experiences (insula, posterior cingulate cortex), reward processing (medial prefrontal cortex, ventral striatum) and cognitive control (dorso-lateral prefrontal cortex, anterior cingulate cortex) [11, 12]. Homeostatic states (hunger versus satiety) and body mass index (BMI) have been associated with the functional connectivity between the hypothalamus, striatum, insula and prefrontal cortex regions [5, 13-16].

\footnotetext{
${ }^{1}$ School of Psychological Sciences and Turner Institute for Brain and Mental Health, Monash University, Melbourne, VIC, Australia. ${ }^{2}$ The Wellcome Centre for Human Neuroimaging, University College London, London, UK. ${ }^{3}$ Department of Electronic Engineering, NED University of Engineering and Technology, Karachi, Sindh, Pakistan. ${ }^{4} \mathrm{CIFAR}$ Azrieli Global Scholars Program, CIFAR, Toronto, ON, Canada. ${ }^{5}$ Department of Neuroscience, Central Clinical School, Monash University, Melbourne, VIC, Australia. ${ }^{6}$ Monash Biomedical Imaging, Monash University, Melbourne, VIC, Australia. ${ }^{7}$ Biomedicine Discovery Institute and Department of Physiology, Monash University, Melbourne, VIC, Australia. email: antonio.verdejo@monash.edu
} 
Specifically, hunger, as compared to satiety, has been associated with increased resting-state connectivity between the insula, the posterior cingulate cortex and the ventromedial prefrontal cortex $[17,18]$. Furthermore, BMI has been associated with increased connectivity between the hypothalamus and the striatum, the insula and the ventromedial prefrontal cortex at rest [19-22], and reduced connectivity between the prefrontal cortex and both the insula and the striatum during food valuation and choice tasks $[11,23]$. These findings variously support different models of obesity. Increased activation of the ventral striatum and ventromedial prefrontal cortex aligns with higher reward sensitivity for food as proposed by the 'hedonic eating' model [7, 24]. Alternatively, lower connectivity between the dorso-lateral prefrontal cortex or anterior cingulate cortex and striatum or ventral medial prefrontal cortex aligns with limitations in cognitive control $[12]$ as proposed by the 'self-regulation' model $[9,10]$. And finally, disrupted connectivity involving the hypothalamus or insula/ posterior cingulate cortex $[17,21,25,26]$ agrees with an 'eating without hunger' framework [8], in which internal homeostatic signals are disregarded. However, a critical limitation of all these studies is that they cannot speak to the direction of information flow between the brain regions (e.g., whether they reflect bottom-up or top-down communication) or the valence of the connections (whether they are excitatory or inhibitory). Such information is needed to reveal if/how, as suggested by neurobiological theories, non-homeostatic brain systems take over homeostatic areas.

Here, we applied a novel hypothesis-testing neural network modelling framework to unravel the direction and valence of connections between hypothalamic, striatal, insula and cortical regions as a function of $\mathrm{BMI}$ and energy homeostasis. We hypothesised that BMI would associate with increased influence of striatal and insula regions on the hypothalamus ('hedonic eating' framework) and decreased influence of cortical regions on lower-level areas (i.e., striatal and insula regions) ('decreased selfregulation' framework). Hunger would be associated with an adaptive increased impact on connectivity in interoceptive regions (insula/pCC), whereas the interaction with $\mathrm{BMI}$ would show a decreased impact on connectivity in interoceptive regions ('eating without hunger' framework).

\section{METHODS \\ Participants}

Forty-one human participants ( 21 females, mean age $=24.37, \mathrm{SD}=5.53$ ) were recruited from the general community. Participants' BMI ranged from 18 to $38 \mathrm{~kg} / \mathrm{m}^{2}$ : 20 had healthy weight $\left(18-24.9 \mathrm{~kg} / \mathrm{m}^{2}\right), 21$ were overweight $\left(\mathrm{BMI}=25-30 \mathrm{~kg} / \mathrm{m}^{2}\right)$ or obese $\left(\mathrm{BMI}>30 \mathrm{~kg} / \mathrm{m}^{2}\right)$ (Table 1 for further descriptive data). An initial screening interview assured that these participants (1) had no history of hypertension or diabetes, (2) had no neurological and psychiatric illness or (3) were on psychoactive medication affecting cognitive functioning or cerebral blood flow. The number of participants was chosen based on a sample size estimation study revealing that 20 participants provided for reliable DCM predictions [27]. In agreement, recent research showed robust model predictions using similar sample sizes when applying spectral dynamic causal modelling (spDCM) to rsfMRI data [28-30]. All participants were naive to the purpose of the study, gave written consent before participating and were reimbursed with $\$ 100$ gift card vouchers. The Monash University Human Research Ethics Committee approved the study (2019-5979-30222) following the Declaration of Helsinki.

\section{Experimental procedure}

Participants completed two fMRI sessions, one after an overnight fast (hunger condition) and one after a standard breakfast (satiety condition). In both conditions, participants were instructed to have a standard meal (700-1000 kj) between $7.30 \mathrm{pm}$ and $8.30 \mathrm{pm}$ on the night prior to their scan, and refrain from eating or drinking (except for water) until their morning scan. For the satiety condition, participants received a standard breakfast ( $293 \mathrm{kcal}$ ) $1 \mathrm{~h}$ prior to their scan. Subjective self-reports of hunger
Table 1. Participants' demographics per BMI group.

\begin{tabular}{|llll|}
\hline & Healthy weight & Overweight & Obese \\
\hline $\begin{array}{l}\text { Age, mean (SD) } \\
\text { Gender (female/ }\end{array}$ & $23.9(5.61)$ & $24.57(1.17)$ & $24.93(6.39)$ \\
$\begin{array}{l}\text { male) } \\
\text { BMI }\left(\mathrm{kg} / \mathrm{m}^{2}\right)\end{array}$ & $21.94(1.94)$ & $3 / 7$ & $8 / 6$ \\
\hline
\end{tabular}

$S D$ standard deviation, $B M I$ body mass index.

( $1=$ not hungry at all; $7=$ very hungry) revealed a significant difference in the perception of hunger between the hunger $(M=4.63 ; S D=1.46)$ and satiety $(M=3.12 ; S D=1.58)$ conditions $(t(40)=4.72, p<0.001)$. There was no significant difference in subjective feelings of fullness across the weight groups when they received a meal $(t(40)=-1.0, p=0.32)$. We therefore did not include subjective feelings of fullness as a parametric regressors into our main spDCM analyses. Both $\mathrm{fMRI}$ sessions were scheduled in the morning between $9 \mathrm{am}$ and $10 \mathrm{am}$ and on average there were 5.82 days $(S D=3.73$ days) between the two scanning sessions. Scheduling the participant's first scan as either part of the hunger or satiety condition was counterbalanced across participants.

Each $\mathrm{fMRI}$ session was divided into an initial task-free fMRI (i.e., restingstate fMRI) sub-session and a subsequent task-based fMRI sub-session. A large body of literature suggests that functional connectivity at rest is altered by previous task experiences (e.g., [31, 32]). As such, task-based fMRI scans were run after the resting-state fMRI scans in order to avoid any artificial inflation of signal-to-noise ratio at rest. The full details of this session and results are outlined in further detail in a previous publication by our group [11], but all relevant sections are repeated here.

Resting-state fMRI sub-session. Resting-state fMRI data were acquired using a 3-Tesla Siemens Skyra MRI scanner equipped with a 32-channel head coil at the Monash Biomedical Imaging Research Centre (Melbourne, Victoria, Australia). During each 8-min scan, 189 gradient-echo planar images comprising of 44 interleaved, continuous axial slices were collected (repetition time $=2500 \mathrm{~ms}$; echo time $=30 \mathrm{~ms}$; flip angle $=90^{\circ} ; 3 \mathrm{~mm}$ isotropic voxels; field of view $=192 \mathrm{~mm}$ ). A whole-brain T1-weighted magnetisation-prepared rapid gradient-echo structural image was also acquired for each session and each participant (192 sagittal slices; $1 \mathrm{~mm}$ isotropic voxels; repletion time $=2300 \mathrm{~ms}$; field of view $=256 \mathrm{~mm}$ ). Participants were instructed to rest while closing their eyes.

Task-based fMRI sub-session. Following the resting-state fMRI scan of each session, participants completed a food choice task in conjunction with functional MRI which results have been previously reported [11]. ROI selection for subsequent spDCM analyses was based on the results revealed during the task-based fMRI session (see details below and Table 2). The food choice task was designed to interrogate the neural basis of food choices using actual physiological stimuli. The task presented pseudorandomised images of pairs of healthy (low sugar and fat) and/or unhealthy (high sugar and fat) beverage combinations in the form healthy-healthy, unhealthy-unhealthy and unhealthy-healthy options. The beverage pair combinations were consistent and presented to all participants in the same order. In each trial, participants were asked to select an option based on their usual preferences. Each image pair was displayed for a 3-s viewing-only period followed by a 1.5-s period where participants made their selection using a two-button response box. The chosen beverage was delivered during the $5 \mathrm{~s}$ following selection. The task comprised five runs, each containing 30 choice events (10 from each condition) and lasting 6 min $32 \mathrm{~s}$.

A computer-controlled MRI-compatible food delivery system (gustometer), consisting of six syringe pumps each connected to a different beverage reservoir, was used to deliver the chosen beverages to participants during simultaneous $\mathrm{fMRI}$ recording. Upon beverage selection, $3 \mathrm{ml}$ of the beverage was delivered via plastic tubing to a mouthpiece mounted on the head coil of the scanner.

The beverages were designed by a professional nutritionist and prepared according to a standard operating procedure. 'Unhealthy' beverages included chocolate, strawberry and caramel milkshakes. 'Healthy' beverages included fruit-blended orange, cranberry/raspberry and veggie juices (including fibre). The unhealthy drinks had significantly more sugar and fat than the healthy drinks: chocolate milkshake $(8.93 \mathrm{~g}$ of fat and $18.99 \mathrm{~g}$ of sugar per $100 \mathrm{ml})$, strawberry milkshake $(8.93 \mathrm{~g}$ of fat and $18.93 \mathrm{~g}$ of sugar per $100 \mathrm{ml})$, caramel milkshake ( $8.93 \mathrm{~g}$ of fat and $18.93 \mathrm{~g}$ of sugar per $100 \mathrm{ml}$ ) versus veggie juice 
Table 2. Locations of regions of interests (spDCM nodes).

\begin{tabular}{llllll} 
Region & Hemisphere & \multicolumn{2}{l}{ MNI coordinates } & Source \\
\cline { 3 - 6 } & & $\boldsymbol{x}$ & $\boldsymbol{y}$ & $\boldsymbol{z}$ & \\
\hline pCC & R & 2 & -42 & 30 & Harding et al. (2018) \\
\hline dIPFC & L & -46 & 42 & 16 & Harding et al. (2018) \\
\hline al & L & -38 & 12 & -6 & Harding et al. (2018) \\
\hline dACC & L & -4 & 32 & 30 & Harding et al. (2018) \\
\hline Hypo & ML & 0 & -3 & -12 & Neurosynth \\
\hline vStr & L & -4 & 8 & -3 & Batra et al. (2013) \\
\hline vmPFC & R & 12 & 58 & 22 & Harding et al. (2018) \\
\hline
\end{tabular}

pCC posterior cingulate cortex, dIPFC dorso-lateral prefrontal cortex, al anterior insula, $d A C C$ dorso-anterior cingulate cortex, Hypo Hypothalamus, vStr ventral striatum, vmPFC ventromedial prefrontal cortex, MNI Montreal Neurological Institute, $R$ right, $L$ left, $M L$ midline.

( $01 \mathrm{~g}$ of fat and $7.7 \mathrm{~g}$ of sugar per $100 \mathrm{ml}$ ), cranberry/raspberry juice (o1 $\mathrm{g}$ of fat and $9.6 \mathrm{~g}$ of sugar per $100 \mathrm{ml}$ ) and orange juice $(01 \mathrm{~g}$ of fat and $8.4 \mathrm{~g}$ of sugar per $100 \mathrm{ml}$ ).

\section{Resting-state fMRI data analyses}

Preprocessing. Functional images were pre-processed for each session separately using SPM12 (revision 12.2, www.fil.ion.ucl.ac.uk). The preprocessing steps consisted of slice time correction, realignment, spatial segmentation and normalisation to the standard EPI template of the Montreal Neurological Institute (MNI) and spatial smoothing using a Gaussian kernel of 8-mm FWHM. No (band-pass) filtering was used except a low-pass filter (of 1/128) that filters the ultra-low frequency scanner drifts [33]. None of the participants exceeded excessive head motion of larger than $3 \mathrm{~mm}$ in translation or 3 mradians rotation.

ROI selection and time series extraction. Seven ROls that are featured in the reviewed neurobiological theories of obesity were identified as key nodes for effective connectivity analyses. The identified neural circuit comprised of the posterior cingulate cortex, dorso-lateral prefrontal cortex, anterior insula, dorso-anterior cingulate cortex, hypothalamus, ventral striatum and ventromedial prefrontal cortex (Fig. 1A). The MNI coordinates for the posterior cingulate cortex, dorso-lateral prefrontal cortex, anterior insula and dorso-anterior cingulate cortex were based on activity associated with food choices during the task-based fMRI session ([11], their Tables 1 and 2). The coordinates for the hypothalamus and ventral striatum were derived from quantitative meta-analyses, as they are part of the food choice network, but were not reported as significant clusters in this sample [11]. The coordinates for the ventral striatum were derived from a coordinate-based meta-analysis of BOLD fMRI experiments examining neural correlates of subjective value [34] and the hypothalamus' coordinates were derived from the Neurosynth (www.neurosynth.com), as no quantitative meta-analysis on (food) decision making and the hypothalamus exists. We did not perform any diagnostic checks in order to check for potential artefacts in the hypothalamus ROI.

To extract BOLD fMRI time series corresponding to the aforementioned ROIs, the pre-processed data were used to establish the residuals of a General Linear Model (GLM). Six head motion parameters and WM/CSF signals were added to the GLM as nuisance regressors. Finally, we selected the $\mathrm{MNI}$ coordinates as the centre of a $6-\mathrm{mm}$ sphere to compute the subject-specific principal eigenvariate and correct for confounds.

\section{Neural network modelling: spectral dynamic causal modelling (spDCM)}

The spDCM analyses were performed using the functions of DCM12 (revision 7196) implemented in SPM12. In order to address our main hypotheses, we focused on spDCM analyses that assessed (1) changes in effective connectivity of hunger versus satiety condition independent of BMI (main effect of hunger), (2) changes in effective connectivity modulated by BMI (main effect of BMI) and (3) changes in hungerrelated effective connectivity modulated by BMI (BMI-by-hunger interaction). Hunger condition was a grouping variable, whereas BMI was a continuous variable. BMI was treated as continuous, as opposed to a grouping variable, as our sample contained overweight $(\mathrm{BMI}=$ $\left.25-30 \mathrm{~kg} / \mathrm{m}^{2}\right)$ and obese $\left(\mathrm{BMI}>30 \mathrm{~kg} / \mathrm{m}^{2}\right)$ individuals.

First-level spDCM analysis. On the first level, a fully connected model was created for each participant and each session (i.e., $7^{2}=49$ connectivity parameters, including seven inhibitory self-connections). Next, we inverted (i.e., estimated) the DCMs using spectral DCM, which fits the complex cross-spectral density using a parameterised power-law model of endogenous neural fluctuations [33]. This analysis provides measures of causal interactions between regions, as well as the amplitude and exponent of endogenous neural fluctuations within each region [33]. Model inversion was based on standard variational Laplace procedures [35]. This Bayesian inference method uses Free Energy as a proxy for (log) model evidence, while optimising the posterior density under Laplace approximation of model parameters.

Second-level spDCM analysis. To characterise how group differences in neural circuitry were modulated by BMI and Hunger condition, hierarchical models over the parameters were specified within a hierarchical Parametric Empirical (PEB) framework for DCM [36]. The three models we used were based on our hypotheses as follows: first, we were interested in the group difference between fasted versus sated conditions and in this PEB analysis, we used BMI, age and gender as regressors of no interest. Second, we were interested associating effective connectivity with BMI and in this PEB analysis, we used group factor (fasted versus sated), age and gender as regressors of interest. Lastly, we were interested in interaction between group factor (fasted versus sated) and BMI and in this PEB analysis, we used BMI, group factor (fasted versus sated), age and gender as regressors of no interest.

Note, that for each of the presented models, all behavioural regressors were mean-centred so that the intercept of each model was interpretable as the mean connectivity. Hunger condition was modelled as the main regressor of interest as a vector consisting of 1 (fasted) and -1 (stated). The interaction term between BMI and Hunger Condition was created by first centreing the continuous variable BMI before creating the elementby-element product of the newly centred BMI variable with the categorical variable Hunger condition [37]. Hunger condition was a grouping variable, whereas BMI was a continuous variable. BMI was treated as continuous, as opposed to a grouping variable, as our sample contained overweight $\left(\mathrm{BMI}=25-30 \mathrm{~kg} / \mathrm{m}^{2}\right)$ and obese $\left(\mathrm{BMI}>30 \mathrm{~kg} / \mathrm{m}^{2}\right)$ individuals.

Bayesian model reduction was used to test all reduced models within each parent PEB model (assuming that a different combination of connections could exist [36] and 'pruning' redundant model parameters); parameters of the best 256 pruned models (in the last Occam's window) were averaged and weighted by their evidence (i.e., Bayesian Model Averaging) to generate final estimates of connection parameters. To identify important effects (i.e., changes in directed connectivity), we compared models (using log Bayesian model evidence to ensure the optimal balance between model complexity and accuracy) with and without each effect and calculated the posterior probability for each model, as a softmax function of the log Bayes factor. We treat effects (i.e., connection strengths and their changes) with posterior probability $>0.99$ as significant for reporting purposes.

Finally, in order to determine the predictive validity (e.g., whether BMI can be predicted from the final, reduced SpDCM's individual connections), leave-one-out cross-validation was performed within the PEB framework [38]. This procedure fits the PEB model to all but one participant and predicts the covariate of interest (e.g., BMI) for the leftout participant. This is repeated with each participant to assess the averaged prediction accuracy for each model. The code is available on reasonable request by contacting the corresponding author. The experiment as it is has been conducted for the first time in our laboratory and no replicates exist yet.

\section{RESULTS}

At the subject level, we used the time series from the ROls to define and estimate a fully connected DCM for each participant and condition using Variational Laplace [35] (Fig. 1A). Post-hoc diagnostic statistics ensured that first-level model inversion had converged (Fig. 1B). The average variance explained across subject-level DCM inversion was very high (Hunger: $M=94.85$, $\mathrm{SD}=1.50$, range $=90.27-97.58$; Satiety: $\mathrm{M}=94.85$, $\mathrm{SD}=1.78$, 

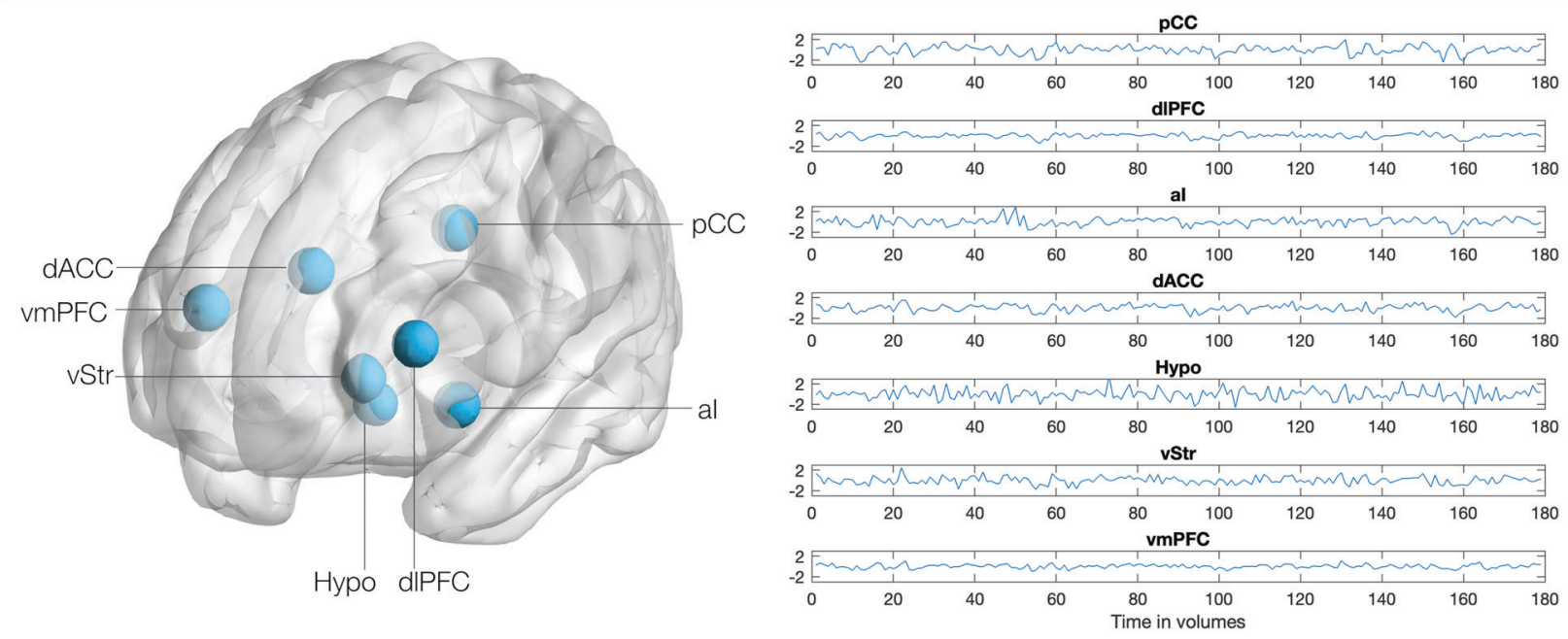

(B) SpDCM diagnostic results per condition
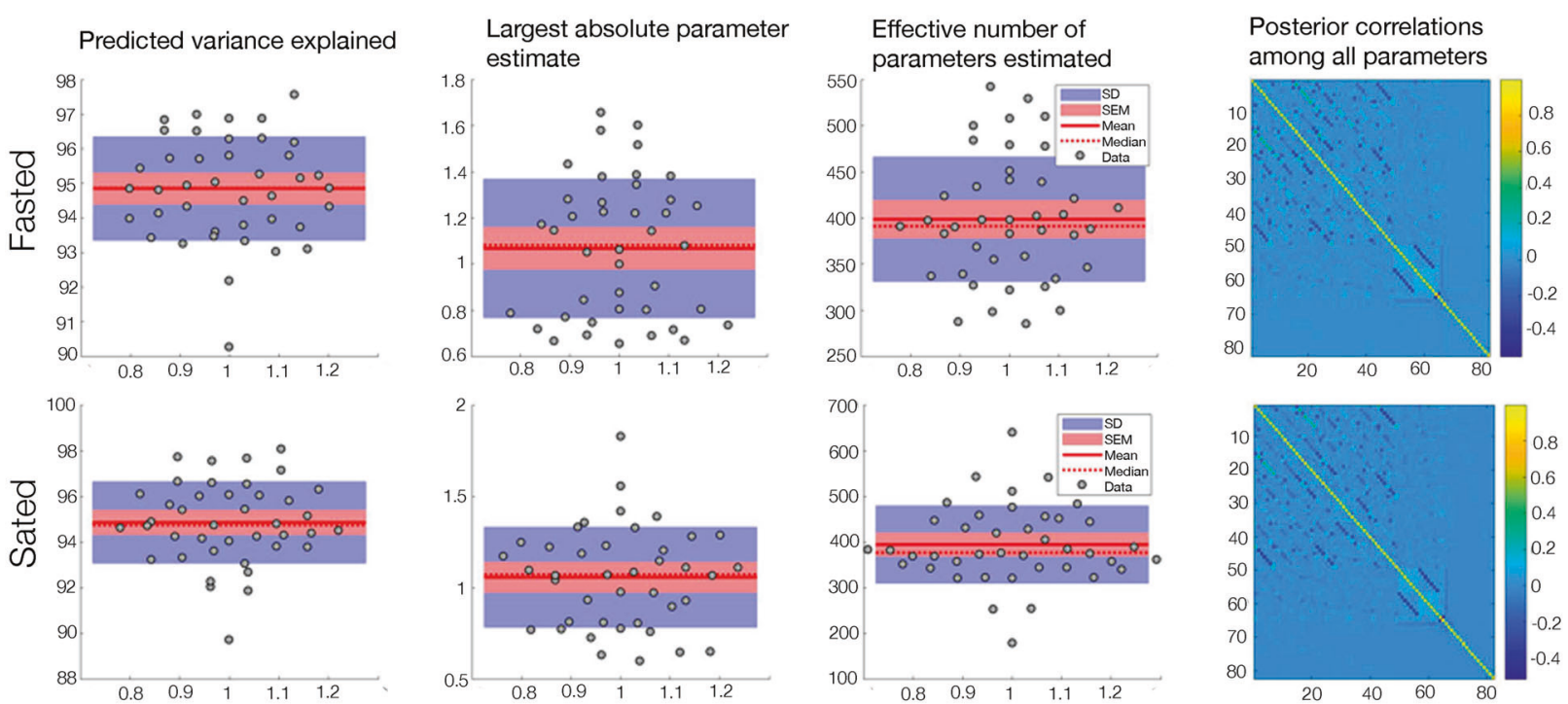

Fig. 1 First-level spDCM modelling in the selected regions of interest. A Brain areas involved in regulated food choice. ROI selection of food choice network was based on task-based fMRI results within the same sample (Harding et al., 2018). (Left) Schematic showing the seven regions of interests (ROIs) used to estimate spDCMs with the fully connected architecture (i.e., $7^{2}=49$ parameter model; Friston et al., 2014 ). (Right) The time series of the ROls for an exemplar subject. B First-level DCM model convergence statistics indicating good model convergence. (First column) Predicted variance explained for each individual was high mostly above $75 \%$. (Second column) The largest absolute parameter estimate did not fall below the typical connection strength of $1 / 8 \mathrm{~Hz}$. (Third column) The effective number of parameters are reported in terms of divergence between the posterior and prior densities over parameters. (Fourth or last column) Posterior correlations among all parameters were low, indicating identifiable parameters.

range $=89.73-98.06$ ), indicating very good model fitting (Fig. 1B, first column). The largest absolute parameter estimate did not fall below the typical connection strength of $1 / 8 \mathrm{~Hz}$ (Hunger: Satiety: $M=1.07, S D=0.30 ;$ Satiety: $M=1.06, S D=0.27$ ) (Fig. $1 B$, second column). The effective number of parameters is reported in terms of divergence between the posterior and prior densities over the parameters (Hunger: $M=398.68, S D=67.52$; Satiety: $M=394.30$; $\mathrm{SD}=84.67$ ) (Fig. 1B, third column). Finally, the posterior correlations among all parameters were low, indicating identifiable parameters (Fig. 1B, final column).

\section{Homeostatic state (hunger versus satiety)}

To explore how homeostatic state is associated with connectivity changes, we examined causal network dynamics during hunger and satiety while controlling for BMI. Starting from a fully connected model (Fig. 1), Bayesian optimisation procedures revealed a sparse model structure with a posterior probability of $>0.99$ at the group level (Fig. 2A and Table 3). Compared to satiety, hunger was associated with an increased excitatory influence of the ventromedial prefrontal cortex over the ventral striatum $(0.14 \mathrm{~Hz}, 95 \% \mathrm{Cl}[0.05,0.23])$ and hypothalamus $(0.26 \mathrm{~Hz}, 95 \% \mathrm{Cl}$ 


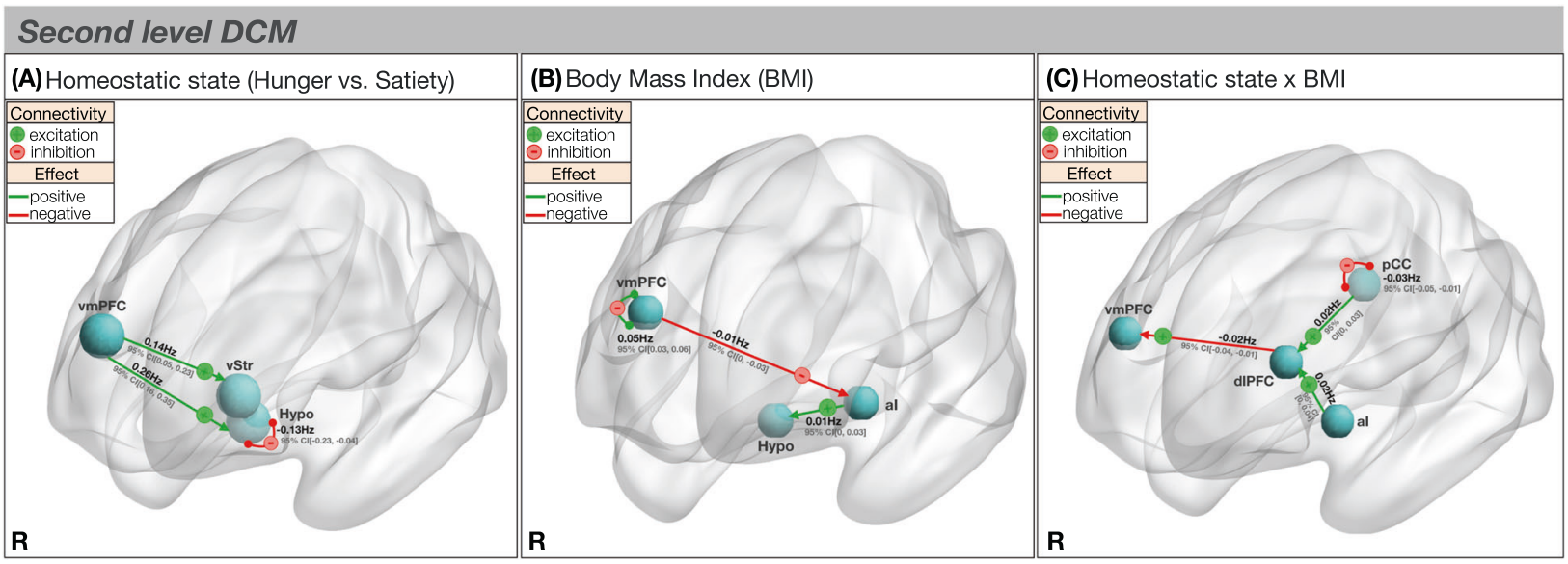

Fig. 2 Second-level spDCM results. A Effective connectivity of hunger. Hunger was associated with increased excitatory connectivity from the ventromedial prefrontal cortex to the ventral striatum and hypothalamus and decreased hypothalamic self-inhibition. B Effective connectivity of BMI. BMI was associated with decreased inhibition from the ventromedial prefrontal cortex to the anterior insula, increased excitation from the anterior insula to the hypothalamus and increased self-inhibition of the ventromedial prefrontal cortex. $\mathbf{C}$ Effective connectivity of BMI $\times$ Hunger State. During hunger, compared to satiety, higher BMI was associated with decreased dorso-lateral prefrontal cortex to ventromedial prefrontal cortex excitation, increased excitation from the posterior cingulate cortex and anterior insula to the dorsolateral prefrontal cortex and decreased posterior cingulate cortex self-inhibition. + or - signs code the parity of connectivity: - inhibitory, + excitatory. pCC posterior cingulate cortex, dIPFC dorso-lateral prefrontal cortex, al anterior insula, Hypo Hypothalamus, vStr ventral striatum, vmPFC ventromedial prefrontal cortex, $\mathrm{R}$ right.

Table 3. Summary of spDCM findings.

\begin{tabular}{|c|c|c|c|c|}
\hline \multicolumn{5}{|c|}{ Homeostatic state (hunger vs. satiety) } \\
\hline Parity & Connection & Hunger vs. satiety effect & Effect size in $\mathrm{Hz}[95 \% \mathrm{Cl}]$ & Posterior probability \\
\hline \multirow[t]{2}{*}{ Excitation } & vmPFC $\rightarrow$ Hypo & + & $0.26[0.16,0.35]$ & 1 \\
\hline & vmPFC $\rightarrow$ vStr & + & $0.14[0.05,0.23]$ & 0.99 \\
\hline Inhibition & Нуро $\rightarrow$ Нуро & - & $-0.13[-0.23,-0.04]$ & 0.99 \\
\hline \multicolumn{5}{|c|}{ Body mass index (BMI) } \\
\hline Parity & & BMI relationship & Effect size in $\mathrm{Hz}[95 \% \mathrm{Cl}]$ & Posterior probability \\
\hline \multirow[t]{4}{*}{ Inhibition } & vmPFC $\rightarrow$ al & - & $-0.01[0,-0.03]$ & 1 \\
\hline & vmPFC $\rightarrow$ vmPFC & - & $-0.05[-0.03,-0.06]$ & 1 \\
\hline & $\mathrm{dACC} \rightarrow \mathrm{dACC}$ & - & $-0.02[0,-0.03]$ & 0.99 \\
\hline & dIPFC $\rightarrow$ dIPFC & - & $-0.02[-0.04,0]$ & 0.99 \\
\hline \multicolumn{5}{|c|}{ Homeostatic state $\times B M I$} \\
\hline Inhibition & $\mathrm{pCC} \rightarrow \mathrm{pCC}$ & - & $-0.03[-0.05,-0.01]$ & 1 \\
\hline
\end{tabular}

$p C C$ posterior cingulate cortex, AIPFC dorso-lateral prefrontal cortex, al anterior insula, dACC dorso-anterior cingulate cortex, Hypo Hypothalamus, vStr ventral striatum, vmPFC ventromedial prefrontal cortex, MNI Montreal Neurological Institute.

$[0.16,0.35])$. We further found less self-inhibition (i.e., disinhibition) of the hypothalamus when individuals were hungry as opposed to sated $(-0.13 \mathrm{~Hz}, 95 \% \mathrm{Cl}[-0.23,-0.04])$. In general, self-inhibition models the recurrent inhibitory activity in the region [39]. Since by definition the self-connections are inhibitory, they suppress activity in the region so as to avoid any run-away excitation in the model. The self-inhibition of the hypothalamus might reflect a suppression of pathways that would normally cause satiety, such as the melanocortin pathway that is known to reduce food intake [40]. Leave-one-out cross-validation revealed that these effects from individual connections are large enough to predict left-out individuals' hunger state above chance level $(r(\mathrm{df}=80)=0.32, p<$ $0.05)$. Cross-validation of this sort provides out of sample estimates of predictability (i.e., the predictive validity of the connectivity strength from a new participant's hunger state).

\section{Body mass index (BMI)}

Next, we explored how BMI is associated with connectivity changes, whilst controlling for homeostatic state. Elevated BMI was associated with an increased excitatory influence of the anterior insula on the hypothalamus $(0.01 \mathrm{~Hz} 95 \% \mathrm{Cl}[0,0.03])$ and a reduced inhibitory influence of the ventromedial prefrontal 
cortex on the anterior insula $(-0.01 \mathrm{~Hz}, 95 \% \mathrm{Cl}[0,-0.03])$ (Fig. $2 \mathrm{~B}$ and Table 3). In addition, individuals with greater BMI had increased self-inhibition of the ventromedial prefrontal cortex $(0.05 \mathrm{~Hz}, 95 \% \mathrm{Cl}[0.03,0.06])$ and dorso-anterior cingulate cortex $(0.02 \mathrm{~Hz}, 95 \% \mathrm{Cl}[0,0.03])$, and decreased self-inhibition of the dorso-lateral prefrontal cortex $(-0.02 \mathrm{~Hz}, 95 \% \mathrm{Cl}[-0.04,0])$ and posterior cingulate cortex $(-0.02 \mathrm{~Hz}, 95 \% \mathrm{Cl}[-0.03,0])$. Selfinhibition of ventromedial prefrontal cortex, dorso-anterior cingulate cortex and dorso-lateral prefrontal cortex associated with increased BMI is likely to reflect different changes in interneuron activity. These differences are to be expected as there are different influences of GABAergic interneurons under these conditions. Leave-one-out cross-validation revealed that these effects sizes from individual connections are large enough to predict group effects with an out of sample estimate $(r(80)=0.22)$.

\section{Interaction of BMI and homeostatic state}

In the final analysis, we investigated how hunger-related connectivity changes may be associated with differences in BMI. During hunger relative to satiety, higher BMI was associated with a lesser excitatory influence of the dorso-lateral prefrontal cortex over the ventromedial prefrontal cortex $(-0.02 \mathrm{~Hz}, 95 \% \mathrm{Cl}[-0.04$, $-0.01])$ and a greater excitatory influence of the anterior insula over the dorso-lateral prefrontal cortex $(0.02 \mathrm{~Hz}, 95 \% \mathrm{Cl}[0,0.04])$ (Fig. 2C and Table 3). In addition, we found decreased selfinhibition of the posterior cingulate cortex $(-0.03 \mathrm{~Hz}, 95 \% \mathrm{Cl}$ $[-0.05,-0.01])$, potentially reflecting different influences of GABAergic interneurons. An increased excitatory influence of the posterior cingulate cortex on the dorso-lateral prefrontal cortex was also evident below the set posterior probability threshold of $>0.99(0.02 \mathrm{~Hz}, 95 \% \mathrm{Cl}[0,0.03]$, posterior probability $=0.94)$. The out of sample correlation between the model's prediction and observed data was significant as revealed by leave-one-out crossvalidation $(r(80)=0.19)$.

\section{DISCUSSION}

This study reveals novel obesity-related changes in directional interactions between corticostriatal and homeostatic regions (summarised in Fig. 3). We specifically examined brain regions featured in neurobiological theories of obesity, including the hedonic eating, eating without hunger and self-regulation views.

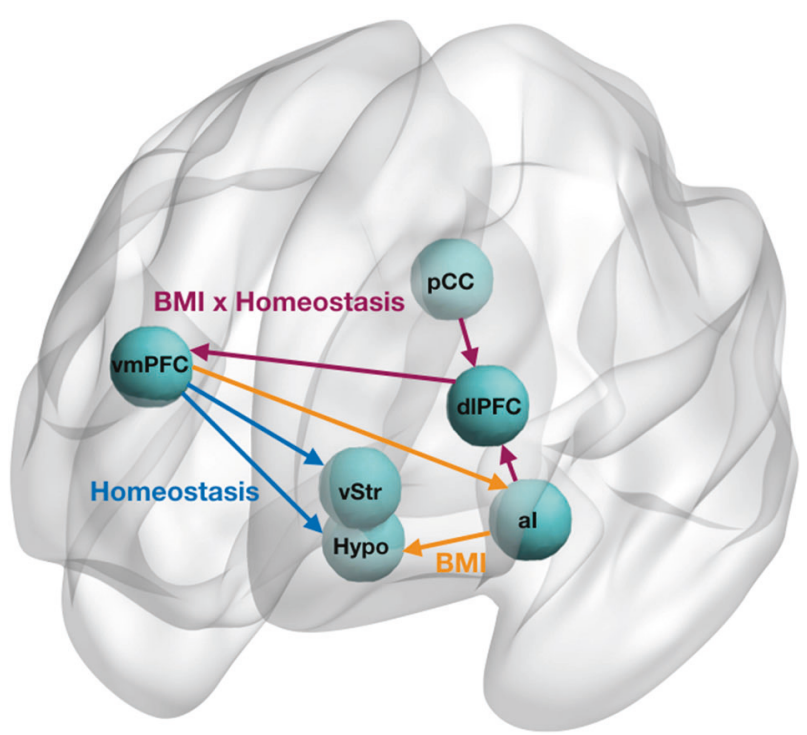

Fig. 3 Overview of spDCM results. Schematic diagram summarising the resting-state neuronal network configurations associated with homeostasis, BMI and homeostasis $\times$ BMI interaction effects.
We found that higher BMI was associated with a greater excitatory influence of the anterior insula on the hypothalamus, regardless of homeostatic state (i.e., during both hunger and satiety). This finding is consistent with reduced sensitivity to changes in energy homeostasis and an eating without hunger view [8-10]. Furthermore, participants with higher BMI showed weaker excitatory influence of the dorso-lateral prefrontal cortex on the ventromedial prefrontal cortex during the hunger state. The interaction between these two regions has been previously associated with dietary self-regulation [12]. In addition, we showed that, regardless of adiposity, during hunger as compared to satiety the ventromedial prefrontal cortex increased its excitatory influence over the ventral striatum and the hypothalamus. This may represent a general adaptive mechanism of top-down signalling during energy deprivation [13].

Together with the hypothalamus, the anterior insula has been proposed to form a homeostatic/interoceptive network. This network prompts eating during energy deprivation and ends feeding upon satiation in humans [17] and plays a central role in food-seeking behaviour in rodents [41]. Our findings suggest that this mechanism is altered in individuals with increased BMI, as increased excitatory input from the insula to the hypothalamus was found irrespective of homeostatic state. This occurs together with reduced inhibition from the ventromedial prefrontal cortex to the anterior insula-a pathway that codes changes in incentive salience in response to changes in homeostatic state [42, 43]. In fitting with this interpretation, increased BMI would be associated with reduced neural sensitivity to changes in homeostatic/ interoceptive state and related persistent attribution of salience to both hunger and satiety states. This argument is consistent with the eating without hunger framework [8], as the hypothalamus (regulating eating based in energy needs) is impacted by systems (i.e., ventromedial prefrontal cortex, anterior insula) encoding nonhomeostatic aspects of food such as incentive salience and hedonic value.

Furthermore, we found that increased BMI was associated with changes in cortico-cortical interactions during the hunger state. Reductions in dorso-lateral prefrontal cortex influence over the ventromedial prefrontal cortex, as observed herein, play a central role in goal-directed food choice tasks and related dietary selfregulation $[12,44]$. The interaction between dorso-lateral prefrontal and the ventromedial prefrontal cortex has been shown to play the key role in context-dependent valuation that requires self-control [45]. However, we cannot assume equivalence between the function of brain regions in task-related versus resting-state designs $[46,47]$, since these regions were activated by a food choice task in the same participants [11]. In the absence of more plausible alternative explanations, we speculate that these findings may relate to alterations in goal-oriented food choice. If our interpretation is correct, these results would align with an impaired self-regulation model of obesity $[9,10]$ but introduce the additional caveat that this mechanism may be statespecific as it was not observed in the satiety state. The increased BMI-related greater excitation from anterior insula and posterior cingulate regions over the dorso-lateral prefrontal cortex is consistent with this hunger-related effect [18].

Communication between 'higher-order' cortical regions and 'lower-order' subcortical areas (e.g., hypothalamus) is critical to governing feeding behaviour [48]. Our results in the hunger (versus satiety) state, irrespective of BMI, support a top-down interpretation of these relationships. This is in line with the notion that hunger triggers an incentive mechanism during energy depletion that motivates food seeking in order to avoid starvation $[49,50]$. Studies in rodent and primate models similarly support the role of ventromedial prefrontal cortex-hypothalamus interactions in feeding behaviour [51]. Notably, we previously reported that homeostatic state influences local activity with the hypothalamus itself [11]. This observation is in line with the established sensitivity of the 
hypothalamus to the neuropeptides that signal and regulate energy needs (e.g., ghrelin and leptin; [52, 53]).

In conclusion, our study reveals changes in directed connectivity between prefrontal, insular, striatal and hypothalamic regions as a function of BMl and homeostatic state. Although the hedonic eating view has been a dominant account of obesity, our findings point to a model in which reduced sensitivity to homeostatic/interoceptive changes and disrupted prefrontal communication during caloric deprivation. Our results highlight the potential for intrinsic predispositions ('neuromarkers'), to improve individualised intervention strategies that can either alter obesogenic traits or ameliorate detrimental effects such as weight gain. They also pave the way for developing non-invasive brain stimulation protocols aimed to rewire cortico-subcortical network abnormalities in obesity. Specifically, our findings provide proof-of-principle evidence to examine the impact of, for example, dorso-lateral prefrontal cortex stimulation and ventromedial prefrontal cortex inhibition on cortical-insularhypothalamic network dynamics and obesity-related therapeutic outcomes. Our study is only the first step to deriving an understanding of this interplay of brain regions at rest; however, it cannot conclusively determine how these resting-state dynamics relate to these networks in action (e.g., feeding behaviour). At this stage, only one study has investigated the relationship between effectivity connectivity at rest and task and their relationship to behaviour [47]. Therefore, complementary future task-based studies will be required to examine how the revealed resting-state network dynamics translate during task performance.

\section{REFERENCES}

1. Grover SA, Kaouache M, Rempel P, Joseph L, Dawes M, Lau DCW, et al. Years of life lost and healthy life-years lost from diabetes and cardiovascular disease in overweight and obese people: a modelling study. Lancet Diabetes Endocrinol. 2015;3:114-22.

2. Tremmel M, Gerdtham U-G, Nilsson P, Saha S. Economic burden of obesity: a systematic literature review. Int J Environ Res Public Health. 2017;14:435.

3. Crino M, Sacks G, Vandevijvere S, Swinburn B, Neal B. The influence on population weight gain and obesity of the macronutrient composition and energy density of the food supply. Curr Obes Rep. 2015;4:1-10.

4. Berthoud H-R. The neurobiology of food intake in an obesogenic environment. Proc Nutr Soc. 2012;71:478-87.

5. Rossi MA, Stuber GD. Overlapping brain circuits for homeostatic and hedonic feeding. Cell Metab. 2018;27:42-56.

6. Berthoud H-R, Münzberg $H$, Morrison $C D$. Blaming the brain for obesity: integration of hedonic and homeostatic mechanisms. Gastroenterology. 2017;152:1728-38.

7. Cameron JD, Chaput J-P, Sjödin AM, Goldfield GS. Brain on fire: incentive salience, hedonic hot spots, dopamine, obesity, and other hunger games. Annu Rev Nutr. 2017;37:183-205.

8. Carnell S, Benson L, Pryor K, Driggin E. Appetitive traits from infancy to adolescence: using behavioral and neural measures to investigate obesity risk. Physiol Behav. 2013;121:79-88.

9. Carter A, Hendrikse J, Lee N, Yücel M, Verdejo-Garcia A, Andrews ZB, et al. The neurobiology of "food addiction" and its implications for obesity treatment and policy. Annu Rev Nutr. 2016;36:105-28.

10. Volkow ND, Wise RA, Baler R. The dopamine motive system: implications for drug and food addiction. Nat Rev Neurosci. 2017;18:741.

11. Harding IH, Andrews ZB, Mata F, Orlandea S, Martínez-Zalacaín I, Soriano-Mas C, et al. Brain substrates of unhealthy versus healthy food choices: influence of homeostatic status and body mass index. Int J Obes. 2018;42:448-54.

12. Hare TA, Camerer CF, Rangel A. Self-control in decision-making involves modulation of the vmPFC valuation system. Science. 2009;324:646.

13. Morton GJ, Meek TH, Schwartz MW. Neurobiology of food intake in health and disease. Nat Rev Neurosci. 2014;15:367-78.

14. Liu S, Borgland SL. Regulation of the mesolimbic dopamine circuit by feeding peptides. Neuroscience. 2015;289:19-42.

15. Cassidy RM, Tong Q. Hunger and satiety gauge reward sensitivity. Front Endocrinol. 2017;8:104. https://doi.org/10.3389/fendo.2017.00104.

16. Livneh Y, Ramesh RN, Burgess CR, Levandowski KM, Madara JC, Fenselau H, et al. Homeostatic circuits selectively gate food cue responses in insular cortex. Nature. 2017;546:611-6.
17. Wright H, Li X, Fallon NB, Crookall R, Giesbrecht T, Thomas A, et al. Differential effects of hunger and satiety on insular cortex and hypothalamic functional connectivity. Eur J Neurosci. 2016;43:1181-9.

18. Al-Zubaidi A, Heldmann M, Mertins A, Brabant G, Nolde JM, Jauch-Chara K, et al. Impact of hunger, satiety and oral glucose on the association between insulin and resting-state human brain activity. FrontHum Neurosci. 2019;13:162. https:// doi.org/10.3389/fnhum.2019.00162.

19. Kilpatrick LA, Coveleskie K, Connolly L, Labus JS, Ebrat B, Stains J, et al. Influence of sucrose ingestion on brainstem and hypothalamic intrinsic oscillations in lean and obese women. Gastroenterology. 2014;146:1212-21.

20. Kullmann S, Heni M, Linder K, Zipfel S, Häring H-U, Veit R, et al. Resting-state functional connectivity of the human hypothalamus: Hypothalamus Functional Connectivity Networks. Hum Brain Mapp. 2014;35:6088-96.

21. Lips MA, Wijngaarden MA, van der Grond J, van Buchem MA, de Groot GH, Rombouts SA, et al. Resting-state functional connectivity of brain regions involved in cognitive control, motivation and reward is enhanced in obese females. Am J Clin Nutr. 2014;100:524-31.

22. Wijngaarden MA, Veer IM, Rombouts SARB, van Buchem MA, Willems van Dijk K, $\mathrm{Pijl} \mathrm{H}$, et al. Obesity is marked by distinct functional connectivity in brain networks involved in food reward and salience. Behav Brain Res. 2015;287:127-34.

23. Verdejo-Román J, Vilar-López R, Navas JF, Soriano-Mas C, Verdejo-García A. Brain reward system's alterations in response to food and monetary stimuli in overweight and obese individuals. Hum Brain Mapp. 2017;38:666-77.

24. Berthoud H-R, Münzberg H, Morrison CD. Blaming the brain for obesity: integration of hedonic and homeostatic mechanisms. Gastroenterology. 2017;152:1728-38.

25. Kilpatrick LA, Coveleskie K, Connolly L, Labus JS, Ebrat B, Stains J, et al. Influence of sucrose ingestion on brainstem and hypothalamic intrinsic oscillations in lean and obese women. Gastroenterology. 2014;146:1212-21.

26. Friston K, Kahan J, Biswal B, Razi A. A DCM for resting state fMRI. Neurolmage. 2014;94:396-407.

27. Goulden N, Elliott R, Suckling J, Williams SR, Deakin JFW, McKie S. Sample size estimation for comparing parameters using dynamic causal modeling. Brain Connect. 2012;2:80-90.

28. Park H-J, Friston KJ, Pae C, Park B, Razi A. Dynamic effective connectivity in resting state fMRI. Neurolmage. 2018;180:594-608.

29. Preller KH, Razi A, Zeidman P, Stämpfli P, Friston KJ, Vollenweider FX. Effective connectivity changes in LSD-induced altered states of consciousness in humans. Proc Natl Acad Sci. 2019;116:2743.

30. Voigt K, Murawski C, Speer S, Bode S. Effective brain connectivity at rest is associated with choice-induced preference formation. Hum Brain Mapp. 2020;41:3077-88. https://doi.org/10.1002/hbm.24999.

31. Tambini A, Ketz N, Davachi L. Enhanced brain correlations during rest are related to memory for recent experiences. Neuron. 2010;65:280-90.

32. Cecchetto C, Fischmeister FPHS, Reichert JL, Bagga D, Schöpf V. When to collect resting-state data: the influence of odor on post-task resting-state connectivity. Neurolmage. 2019;191:361-6.

33. Razi A, Kahan J, Rees G, Friston KJ. Construct validation of a DCM for resting state fMRI. Neurolmage. 2015;106:1-14.

34. Bartra O, McGuire JT, Kable JW. The valuation system: a coordinate-based metaanalysis of BOLD fMRI experiments examining neural correlates of subjective value. Neurolmage. 2013;76:412-27.

35. Friston K, Mattout J, Trujillo-Barreto N, Ashburner J, Penny W. Variational free energy and the Laplace approximation. Neurolmage. 2007;34:220-34.

36. Friston KJ, Litvak V, Oswal A, Razi A, Stephan KE, van Wijk BCM, et al. Bayesian model reduction and empirical Bayes for group (DCM) studies. Neurolmage. 2016;128:413-31.

37. Aiken LS, West SG. Multiple regression: testing and interpreting interactions. Thousand Oaks, CA, US: Sage Publications, Inc; 1991.

38. Zeidman $P$, Jafarian A, Seghier ML, Litvak V, Cagnan $H$, Price $C J$, et al. A guide to group effective connectivity analysis, part 2: second level analysis with PEB. Neurolmage. 2019;200:12-25.

39. Friston K, Harrison L, Penny W. Dynamic causal modelling. Neurolmage. 2003;19:97-109.

40. Baldini G, Phelan KD. The melanocortin pathway and control of appetite-progress and therapeutic implications. J Endocrinol. 2019;241:R1-R33.

41. Kusumoto-Yoshida I, Liu H, Chen BT, Fontanini A, Bonci A. Central role for the insular cortex in mediating conditioned responses to anticipatory cues. Proc Natl Acad Sci. 2015;112:1190.

42. Damasio AR, Tranel D, Damasion H. Somatic markers and the guidance of behaviour: theory and preliminary testing. In: Stuss DT and Knight RT, editors. Frontal lobe function and dysfunction. New York: Oxford UP; 1991. p. 217-29.

43. Verdejo-García A, Bechara A. A somatic marker theory of addiction. Neuropharmacology. 2009;56:48-62.

44. Hare TA, Malmaud J, Rangel A. Focusing attention on the health aspects of foods changes value signals in vmPFC and improves dietary choice. J Neurosci. 2011;31:11077-87. 
45. Rudorf S, Hare TA. Interactions between dorsolateral and ventromedial prefrontal cortex underlie context-dependent stimulus valuation in goal-directed choice. J Neurosci Off J Soc Neurosci. 2014;34:15988-96.

46. Poldrack R. Can cognitive processes be inferred from neuroimaging data? Trends Cogn Sci. 2006;10:59-63.

47. Jung K, Friston KJ, Pae C, Choi HH, Tak S, Choi YK, et al. Effective connectivity during working memory and resting states: a DCM study. Neurolmage. 2018;169:485-95.

48. Andermann ML, Lowell BB. Toward a wiring diagram understanding of appetite control. Neuron. 2017;95:757-78.

49. Betley JN, Xu S, Cao ZFH, Gong R, Magnus CJ, Yu Y, et al. Neurons for hunger and thirst transmit a negative-valence teaching signal. Nature. 2015;521:180.

50. Krashes MJ, Koda S, Ye C, Rogan SC, Adams AC, Cusher DS, et al. Rapid, reversible activation of AgRP neurons drives feeding behavior in mice. J Clin Invest. 2011;121:1424-8.

51. Ongur D, Price JC. The organization of networks within the orbital and medial prefrontal cortex of rats, monkeys and humans. Cereb Cortex. 2000;10:206-19.

52. Farooqi IS, Bullmore E, Keogh J, Gillard J, O'Rahilly S, Fletcher PC. Leptin regulates striatal regions and human eating behavior. Science. 2007;317:1355.

53. Malik S, McGlone F, Bedrossian D, Dagher A. Ghrelin modulates brain activity in areas that control appetitive behavior. Cell Metab. 2008;7:400-9.

\section{ACKNOWLEDGEMENTS}

The authors thank Richard McIntyre for help with MRI data acquisition. This study was supported by an Australian National Health and Medical Research Council (NHMRC) grant (1140197) granted to AV-G. AV-G is supported by a CDF fellowship from the Australian Medical Research Future Fund (MRF1141214).

\section{AUTHOR CONTRIBUTIONS}

All authors conceptualised the study design. KV and AR analysed the data. KV wrote initial manuscript draft. All authors contributed to writing and revising the manuscript.
COMPETING INTERESTS

The authors declare no competing interests.

\section{ADDITIONAL INFORMATION}

Supplementary information The online version contains supplementary material available at https://doi.org/10.1038/s41366-021-00918-y.

Correspondence and requests for materials should be addressed to A.V.-G.

Reprints and permission information is available at http://www.nature.com/ reprints

Publisher's note Springer Nature remains neutral with regard to jurisdictional claims in published maps and institutional affiliations.

(c) (i)

Open Access This article is licensed under a Creative Commons Attribution 4.0 International License, which permits use, sharing, adaptation, distribution and reproduction in any medium or format, as long as you give appropriate credit to the original author(s) and the source, provide a link to the Creative Commons license, and indicate if changes were made. The images or other third party material in this article are included in the article's Creative Commons license, unless indicated otherwise in a credit line to the material. If material is not included in the article's Creative Commons license and your intended use is not permitted by statutory regulation or exceeds the permitted use, you will need to obtain permission directly from the copyright holder. To view a copy of this license, visit http://creativecommons. org/licenses/by/4.0/.

(c) The Author(s), under exclusive licence to Springer Nature Limited 2021 